\title{
Travaux Pratiques autour d'une Chaîne de Mesure (de température) pour un Public de Licence Professionnelle Orientée Agroalimentaire
}

\author{
Jérôme MATHIEU ${ }^{1,2}$ \\ ${ }^{1}$ LAIN, Univ. Montpellier II, P1. E. Bataillon, CC 082, 34095 MONTPELLIER Cedex 5, mathieu@lain.univ-montp2.fr \\ ${ }^{2}$ IUT de Rodez pour CUFR J.F. Champollion, av. Europe, BP 3219, 12032 RODEZ Cedex 9
}

\begin{abstract}
RESUME Ces travaux pratiques sont donnés aux étudiants de la Licence Professionnelle «Gestion des mesures, contrôles et capteurs dans les industries agroalimentaires ». La formation et le profil des étudiants sont présentés. Ces travaux pratiques s'insèrent dans un ensemble de cours / TD / TP relatifs aux chaînes de mesure. Ils ont pour objectif de préparer les étudiants au traitement global d'une chaîne de mesure, du montage à son utilisation en passant par son réglage et son étude métrologique. Pour ces travaux pratiques, on envisage un découpage de l'étude des chaînes de mesure par rapport au contexte d'utilisation : celui du laboratoire, où les appareils manuels sont prépondérants, et celui du site de production où les chaînes de mesure sont pilotées par des automates industriels. Ces deux contextes correspondent à deux travaux pratiques menés coup sur coup de manière à faire le parallèle et à faire ressortir les différences. Chaque TP est découpé en 4 parties : présentation, choix des éléments et montage de la chaîne de mesure ; réglage (calibrage) de la chaîne ; étude métrologique de la chaîne ; et utilisation de la chaîne dans un contexte métrologique (étalonnage ou vérification). Les chaînes étudiées sont des chaînes de mesure de température. La conclusion récapitule les intérêts qu'offre ces travaux pratiques dans la compréhension de l'ensemble du cours.
\end{abstract}

Mots clés : chaîne de mesure, température, travaux pratiques, convertisseurs de température, API.

\section{INTRODUCTION}

\subsection{Présentation de la Formation}

Le Centre Universitaire de Formation et de Recherche (CUFR) Jean-François Champollion (www.univ-jfc.fr) propose sur le site de Rodez une Licence Professionnelle intitulée " Gestion des mesures, contrôles et capteurs dans les industries agroalimentaires » (www.univ-jfc.fr/f_lipro/lipro_gestcapteurs.php).

Le public visé par cette formation est très varié. Certains étudiants ont déjà une culture EEA, d'autres non et ont plutôt une culture agroalimentaire ou biotechnologique.

Parmi les notions abordées par la formation liées à l'EEA, sont abordées la métrologie, l'instrumentation et les capteurs, la commande des systèmes de production et le suivi de production.

\subsection{Contexte des Travaux Pratiques}

Naturellement, la chaîne de mesure est un point clé de la formation. Nous avons opté pour une présentation destinée à tout public.

Cette notion de chaîne de mesure est d'abord appréhendée en cours par une décomposition en briques élémentaires (transducteur, conditionneur, transmetteur, CAN, ...) permettant le passage du mesurande au résultat de mesurage. Chaque brique fait l'objet d'une description essentiellement conceptuelle (rôles) et assez peu technique. Du point de vue EEA, on ne s'attache qu'à décrire les grandes fonctions électroniques liées aux chaînes de mesure: conditionnement, amplification, isolation, conversion analogique / numérique, ... Chaque fonction est schématisée en nommant les principaux composants électroniques réalisant la fonction, mais sans entrer dans les détails des architectures électroniques et de leur fonctionnement.

Des travaux dirigés viennent compléter cette première approche par une analyse de documents techniques de différentes références (capteur, convertisseur, instrument, ...) afin d'entraîner l'étudiant à identifier les différentes briques. L'identification permet de :

- comprendre la place de la référence dans une chaîne de mesure complète ;

- trouver les références à ajouter pour finaliser une chaîne de mesure ;

- déterminer les paramètres importants d'une fiche technique par rapport aux rôles de la référence ;

- déjouer certaines techniques marketing ;

- etc.

L'étape suivante, l'objet de l'article, consiste, sous forme de travaux pratiques, à préparer l'étudiant au traitement global d'une chaîne de mesure. Il correspond au montage, au réglage ou calibration, à l'étude métrologique et enfin à l'utilisation de la chaîne de mesure. Cette présentation globale permet d'offrir aux étudiants du recul, une vision plus générale de la chaîne de mesure. Elle permet aussi une mise en perspective des différents cours et TD traitant du sujet.

Schématiquement, les chaînes de mesure sont utilisées dans deux principaux contextes différents : le laboratoire et le site de production. Ce découpage est important car, en pratique, en découle une différence de constitution des chaînes de mesure. Les briques élémentaires ne se présentent pas sous les mêmes formes. On effectue donc deux travaux pratiques avec le même type de chaîne de mesure : ici pour mesurer la température. Chaque TP est sensé être représentatif du contexte de référence. Afin de complexifier le travail des étudiants, de diversifier les matériels utilisés et de pouvoir 
effectuer des comparaisons, les chaînes de mesure dans les deux TPs ne sont pas strictement identiques.

\subsection{Déroulement des Travaux Pratiques}

Chaque TP se décompose en quatre étapes :

- montage de la chaîne de mesure à partir d'un ensemble de matériels disponibles répondant ou non aux besoins. Cette étape est une application directe des travaux dirigés. Elle implique la compréhension du rôle de chaque brique dans la production et l'acheminement de l'information du mesurande jusqu'au résultat de mesurage exploitable. Elle impose une lecture approfondie des documentations techniques ;

- calibrage de la chaîne de mesure. Cette étape implique la compréhension des interactions de chaque brique dans la production du résultat de mesurage exploitable. Peu d'informations mais plus de réflexion sont nécessaires ;

- $\quad$ étude métrologique de la chaîne de mesure ;

- utilisation de la chaîne de mesure dans un but métrologique d'étalonnage ou de vérification.

Les deux dernières étapes font plutôt référence aux cours et TD de métrologie.

Par la suite, chaque TP fait l'objet d'un paragraphe distinct dans lequel sont développées les différentes étapes au travers de différents sous-paragraphes.

\subsection{A propos de la Notion de Capteur}

Dans la pratique, les termes normalisés [1] sont rarement employés à bon escient. Il me semble que le terme de «capteur» et ces multiples synonymes (sonde, détecteur, palpeur, ...) ne signifient plus grand chose d'autre qu'une boîte noire délivrant une valeur ou un signal faisant office de résultat de mesurage. Je préfère donc utiliser le terme de «transducteur » pour nommer la partie sensible (avec éventuellement le corps d'épreuve) c'est-à-dire ce qui correspond au « capteur» dans [1] (éventuellement composite [2]). J'utilise le terme de « capteur » pour nommer l'objet, la boîte noire.

Cette « permutation » des définitions entre « capteur » et «transducteur» pourrait perturber les étudiants. Toutefois, ces choix sont expliqués en cours. Et il me semble que cela permet de lever bien des confusions lorsqu'il s'agit d'analyser des documentations techniques notamment.

\section{TRAVAUX PRATIQUES DANS UN CONTEXTE DE LABORATOIRE}

Dans le cadre du laboratoire, nous avons choisi une solution assez simple, modulaire, économique et manuelle constituée de :

- transducteur : résistance Pt 1002 fils ;

- conditionnement du signal et transmetteur : convertisseur de température Brodersen modèle PXT-10-230 ;

- multimètre analogique.
Pourquoi ces choix? Nous avons pu constater lors de visites d'entreprise dans la branche agroalimentaire que leurs laboratoires étaient équipés d'instruments de mesure. Ces instruments sont simples d'emploi. Mais pédagogiquement, ils n'apportent pas grand chose puisque l'intégralité de la chaîne de mesure est cachée à l'utilisateur. Nous utilisons toutefois un tel appareil dans le paragraphe 3.5 afin de montrer aux étudiants les avantages et inconvénients par rapport à une chaîne de mesure « éclatée ».

\subsection{Montage de la Chaîne de Mesure}

Les étudiants doivent choisir les éléments pour répondre à la demande et faire le montage proprement dit. Le montage consiste à relier électriquement les différents éléments de la chaîne (cf. figure 1). Le convertisseur demande en plus une configuration à l'aide de shunts entre différents connecteurs. Ensuite, on branche les différentes alimentations électriques.

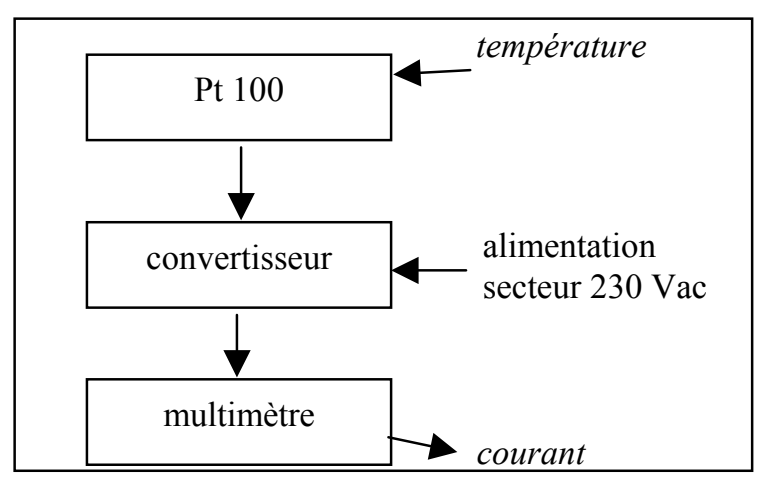

fig 1 : vue globale et modulaire de la chaîne de mesure de la température par Pt 100

Au niveau du conditionneur de signal, les étudiants ont accès à la documentation technique fournie par le fabricant, documentation où figure le schéma électronique suivant :

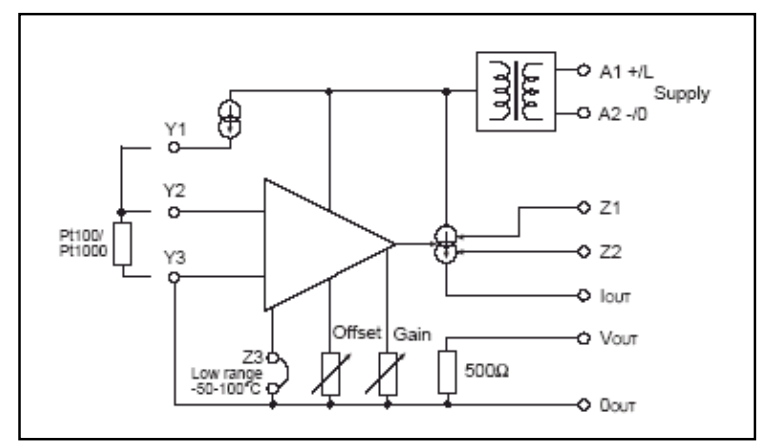

fig 2 : schéma électronique du convertisseur de température pour Pt 100 (Brodersen PXT-10-230)

Compte tenu du matériel dont nous disposons, les étudiants doivent réaliser les câblages suivants :

- $\quad$ le transducteur est connecté à Y2 et Y3 ;

- un shunt est nécessaire entre Y1 et Y2 car le conditionneur est prévu pour fonctionner avec des transducteurs 3 fils ; 
- un shunt est réalisé entre $\mathrm{Y} 3$ et $\mathrm{Z} 3$ pour limiter l'étendue de mesure de $-50{ }^{\circ} \mathrm{C}$ à $+100{ }^{\circ} \mathrm{C}$;

- le convertisseur autorise plusieurs types de sortie : en tension ou en courant. Pour diversifier les pratiques, nous imposons ici l'utilisation de la sortie courant 4-20 mA puisque seule la tension est utilisable dans le TP donné au paragraphe 4. Le multimètre, avec un calibre $50 \mathrm{~mA}$, est alors connecté à Iout $(+)$ et 0out (masse) ;

- le convertisseur dispose d'un transformateur lui permettant d'être directement alimenté en 230 Vac. Cette alimentation est connectée à A1 et A2. Il s'agit ici d'être extrêmement prudent car les connecteurs n'offrent pas de protection aux électrocutions suffisante.

Une thermorésistance comme la Pt100 étant un transducteur passif, il est nécessaire d'apporter une source électrique pour obtenir un signal électrique. D'après le montage proposé (figure 2), la source est ici une source de courant. On constate également que le shunt entre $\mathrm{Y} 1$ et $\mathrm{Y} 2$ est indispensable car la source est reliée à $\mathrm{Y} 1$ et non à $\mathrm{Y} 2$.

Dans le schéma figure 2, on reconnaît un transformateur pour convertir le $230 \mathrm{Vac}$ en une tension continue pour les sources de courant.

Aux bornes du transducteur, symbolisé par une résistance, on recueille une tension qui est ensuite amplifiée. L'amplificateur est relié à deux résistances ajustables (potentiomètres) qui permettent de régler le gain et l'offset. Ce point est intéressant et est déjà abordé en cours. Les phénomènes physiques utilisés dans les transducteurs obéissent en général à des lois nonlinéaires. Cependant, pour des raisons évidentes ici de simplicité de mise en œuvre, les lois de conversion mesurande en grandeur électrique - sont approximées par des lois affines du type $y=a \cdot x+b$ où $a$ est le gain et $b$ l'offset. Il en résulte deux conséquences métrologiques :

- une limitation des étendues de mesure pour limiter les écarts entre la loi physique et l'approximation linéaire ;

- une source d'erreurs systématiques due à ces écarts.

Les différences entre un Pt 100 avec un montage 2 fils, 3 fils ou 4 fils sont abordées uniquement en terme de montage et de qualité métrologique (précision, linéarité, dérive, ...).

\subsection{Calibrage de la Chaîne de Mesure}

Deux opérations sont importantes dans ce cadre.

La première consiste à déterminer théoriquement la loi affine qui relie le courant mesuré par le multimètre à la température détectée par le transducteur.

Soit $y$ la température $\left({ }^{\circ} \mathrm{C}\right), x$ le courant mesuré $(\mathrm{mA}), a$ le gain théorique $\left({ }^{\circ} \mathrm{C} / \mathrm{mA}\right)$ et $b$ l'offset théorique $\left({ }^{\circ} \mathrm{C}\right)$. L'étendue de mesure est donnée de $-50^{\circ} \mathrm{C}$ à $+100^{\circ} \mathrm{C}$ (cf. § 2.1). Le convertisseur de température est utilisé avec une sortie en courant en 4-20 mA (cf. $\S 2.1$ ). Donc $-50{ }^{\circ} \mathrm{C}$ correspond à $4 \mathrm{~mA}$ et $+100^{\circ} \mathrm{C}$ correspond à $20 \mathrm{~mA}$. Cela donne le système de 2 équations suivant :

$$
\left\{\begin{aligned}
-50 & =4 a+b \\
+100 & =20 a+b
\end{aligned}\right.
$$

soit $a=9,375{ }^{\circ} \mathrm{C} / \mathrm{mA}$ et $b=-87,5{ }^{\circ} \mathrm{C}$.

D'où la loi affine : $y=9,375 x-87,5$.

La deuxième opération consiste à régler la chaîne de mesure de manière à pouvoir retrouver expérimentalement la loi linéaire déterminée théoriquement cidessus. Ici, seul le convertisseur nécessite un réglage au niveau des deux paramètres que sont le gain et l'offset. Le fabricant du convertisseur ne préconise pas de méthode particulière. Un étalonnage à l'aide d'un bain thermostaté correspond à la solution retenue. Cette opération est décrite au paragraphe 2.4.

\subsection{Etude Métrologique de la Chaîne de Mesure}

L'analyse métrologique porte uniquement sur la détermination de l'incertitude-type de l'ensemble de la chaîne de mesure à partir des données fournies par les fabricants dans les documentations. Il s'agit donc d'une incertitude-type de type B.

La première étape consiste à relever toutes les sources d'erreur connues :

- précision du transducteur Pt 100 : classe B (IEC 751) c'est-à-dire d'une précision de $\pm(0,3+0,5 \% \mid T){ }^{\circ} \mathrm{C}$ où $T$ est la température ;

- précision du convertisseur : $1 \%$ (précision) et $0,05 \%$ de la pleine échelle (linéarité). Influence du coefficient de température négligé ;

- précision du multimètre : $2,5 \%$ de la pleine échelle (calibre $50 \mathrm{~mA}$ );

- erreur de lecture sur le multimètre analogique : graduation $1 \mathrm{~mA}$.

Certaines erreurs donnent des incertitudes qui s'expriment ici en ampère. La loi de propagation des erreurs appliquée à l'équation liant la température au courant mesuré par le multimètre (cf. paragraphe 2.2) convertit les incertitudes exprimées en ampère en incertitudes exprimées en degré Celsius.

On obtient au bout du compte, à température ambiante $\left(22^{\circ} \mathrm{C}\right)$, une incertitude-type de type $\mathrm{B}$ de $13{ }^{\circ} \mathrm{C}$. Cette valeur est importante et limite fortement l'utilisation de cette chaîne de mesure, notamment en regard de son étendue de mesure.

Compte tenu du matériel à notre disposition, le multimètre constitue la principale source d'erreurs. Elle représente pratiquement $90 \%$ de l'erreur totale. L'erreur de lecture représente à elle seule plus de $40 \%$ de l'erreur totale. Le convertisseur est à l'origine de moins de $10 \%$ de l'erreur totale. Enfin, l'erreur due au transducteur est négligeable (environ $2 \%$ ).

Cette petite analyse permet d'apporter des éclaircissements ou de retrouver des remarques données dans le cours. Les mesurages avec des transducteurs du type $\mathrm{Pt}$ 
100 sont relativement précises $\left(1,5^{\circ} \mathrm{C}\right.$ environ $)$ à condition de pouvoir lire précisément le courant sortant du convertisseur. Les mesurages sont également peu sensibles à la qualité des calibrages. En effet, la précision retenue pour le convertisseur correspond à la pire situation et n'engendre malgré tout que $10 \%$ d'erreur. Par conséquent, l'attention de l'acheteur doit surtout porter sur le choix du multimètre.

\subsection{Utilisation de la Chaîne de Mesure : Etalonnage} à l'Aide d'un Bain Thermostaté

On dispose d'un bain thermostaté avec un capteur de température intégré étalonné à $0,1^{\circ} \mathrm{C}$ près. Le transducteur est plongé dans ce bain.

La démarche proposée est la suivante :

- On impose à l'eau du bain une température TO correspondant à une température proche d'une extrémité de l'étendue de mesure choisie. On lit $x 0$ sur le multimètre ;

- On impose à l'eau du bain une nouvelle température $T 1$ correspondant à une température proche de l'autre extrémité de l'étendue de mesure choisie. On lit $x 1$ sur le multimètre;

- On règle le gain de manière à modifier le courant lu sur le multimètre de la valeur initiale $x 1$ jusqu'à obtenir $x^{\prime}=\frac{T 1-T 0}{a}+x 0$. Cette opération permet de régler le gain pour obtenir la valeur théorique $a$ calculée au paragraphe 2.2 ;

- On règle ensuite l'offset de manière à modifier le courant lu sur le multimètre de la valeur $x$ ' jusqu'à obtenir $x=\frac{T 1-b}{a}$. Cette opération permet de régler l'offset pour obtenir la valeur théorique $b$ calculée au paragraphe 2.2.

\section{TRAVAUX PRATIQUES DANS UN CONTEXTE DE SITE DE PRODUCTION}

Dans le cadre d'un site de production, nous avons choisi une solution orientée vers les automates industriels. Cette solution est constituée de :

- transducteur : thermocouple type K ;

- conditionnement du signal et transmetteur: convertisseur Phoenix-Contact modèle MCRTE-JK-U-E ;

- API Siemens LOGO ! 24 + câble API - PC + logiciel LOGO ! Soft Comfort V 4.0

Pourquoi ces choix ? Le convertisseur est prévu pour être monté avec un API. L'utilisation d'un thermocouple permet de montrer les apparentes similitudes dans les montages et les différences fondamentales dans le fonctionnement des chaînes de mesure basées sur un transducteur passif (première série de travaux pratiques) ou basées sur un transducteur actif (présente série de travaux pratiques).

L'API Siemens LOGO ! 24 dispose de 2 entrées mixtes (analogiques ou numériques) qui permettent de connecter directement le convertisseur à l'API. Il dispose également d'une alimentation stabilisée en $24 \mathrm{~V}$ continu utilisable pour les autres composants de la chaîne de mesure. Enfin, cet API est fourni avec un câble et un logiciel permettant de le programmer et d'observer l'évolution du programme « en temps réel » depuis un ordinateur PC. Un clavier et un écran sur l'API permettent d'effectuer si besoin les mêmes opérations qu'avec le logiciel mais avec moins de confort.

\subsection{Montage de la Chaîne de Mesure}

Une fois encore, les étudiants doivent choisir les éléments pour répondre à la demande et faire le montage proprement dit. Le montage consiste à relier électriquement les différents éléments de la chaîne (schéma similaire à la figure 1). Le convertisseur demande en plus une configuration par commutateurs DIP. Ensuite, on branche les différentes alimentations électriques.

Au niveau du convertisseur, les étudiants ont accès à la documentation technique fournie par le fabricant, documentation où figure le schéma électronique suivant :

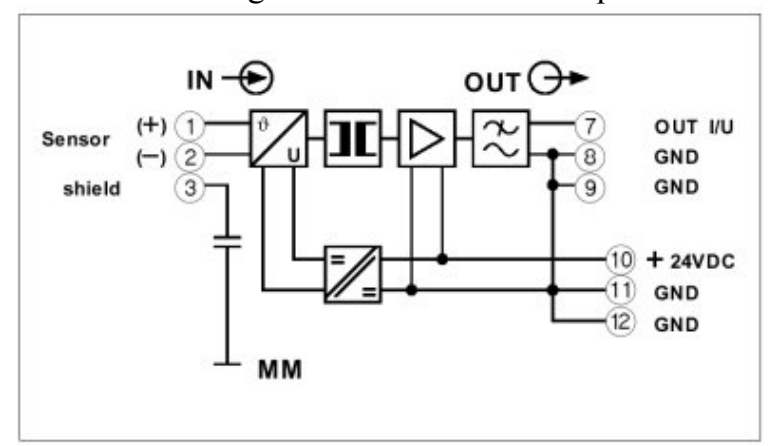

fig 3 : schéma-bloc électronique du convertisseur de température pour thermocouple (Phoenix-Contact MCR)

Compte tenu du matériel dont nous disposons, les étudiants doivent réaliser les câblages suivants :

- le transducteur est connecté aux bornes 1 et 2 ;

- l'entrée analogique de l'API est branchée à la borne 7 et sa masse à la borne 8 ;

- $\quad$ le $\oplus$ de l'alimentation de l'API est branchée à la borne 10 et sa masse à la borne 11 .

Le convertisseur autorise plusieurs modes de fonctionnement en fonction des commutateurs DIP suivants :

\begin{tabular}{|c|c|c|c|c|c|c|c|}
\hline 1 & 2 & 3 & 4 & 5 & 6 & 7 & 8 \\
\hline Off & Off & Off & Off & On & On & Off & Off \\
\hline $\begin{array}{c}\text { type } \\
\mathrm{K}\end{array}$ & \multicolumn{2}{|c|}{$\begin{array}{c}\text { plage de } \mathrm{t}^{\circ} \mathrm{C} \\
0-400^{\circ} \mathrm{C}\end{array}$} & \multicolumn{2}{|c|}{$\begin{array}{c}\text { soudure } \\
\text { froide à } \\
\text { compenser }\end{array}$} & $\begin{array}{c}\text { sortie } \\
0-10 \mathrm{~V}\end{array}$ \\
\hline
\end{tabular}

La première ligne indique le numéro du commutateur DIP, la deuxième ligne indique l'état du commutateur et la troisième ligne commente les réglages choisis. Les étudiants doivent définir l'état de chaque commutateur DIP à partir de choix imposés par l'énoncé énoncé rédigé comme les commentaires du tableau - et à partir de la documentation du fabricant. 
Le convertisseur accepte deux types de thermocouple, $\mathrm{J}$ et $\mathrm{K}$.

L'analyse du schéma-bloc de la figure 3 est intéressante surtout en comparaison entre la théorie (le cours) et la pratique (ce TP). En prenant les blocs de gauche à droite, de haut en bas :

- le premier bloc convertit la température $\theta$ en tension continue U. Il symbolise la compensation automatique de la soudure froide proposée par le convertisseur (commutateurs DIP 5 et 6 à Off). En effet, la compensation de la soudure froide revient à ajouter une f.é.m., fonction de la température du convertisseur et donc de la soudure froide. On n'utilise pas cette fonction dans ce TP car on perd en précision ;

- le thermocouple est isolé galvaniquement pour protéger le convertisseur ;

- l'amplification assure le passage des $\mathrm{mV}$ fournis par le thermocouple (transducteur actif) aux V utilisés pour la transmission (transmetteur en mode $0-10 \mathrm{~V}$ ). Le gain et l'offset sont réglables (même si cela n'apparaît pas dans la figure 3) lorsque la compensation automatique de la soudure froide n'est pas utilisée (commutateurs DIP 5 et 6 à On). Contrairement au TP précédent, l'amplification n'assure pas la conversion température en tension ou courant, puisque ici nous avons affaire à un transducteur actif, mais plutôt le rôle de transmetteur. Comme au précédent TP, le convertisseur est construit pour produire une loi affine entre la température et la tension délivrée par le convertisseur, avec les conséquences déjà décrites au paragraphe 2.1. Seul un traitement logique au niveau de l'API pourrait corriger ;

- un filtre analogique passe-bas termine cette première ligne. Il assure un lissage pour éliminer le bruit principalement issu du thermocouple ;

- en deuxième ligne, l'unique bloc est un convertisseur de tension continue pour adapter le $24 \mathrm{~V}$ fournit aux bornes 10 et 11 en une tension utilisable par l'électronique du convertisseur.

\subsection{Réglage de la Chaîne de Mesure}

Comme pour le TP précédent, deux opérations sont importantes et seul le convertisseur nécessite un calibrage.

La loi affine relie la tension relevée par l'API à la température détectée par le transducteur.

Soit $y$ la température $\left({ }^{\circ} \mathrm{C}\right), x$ la tension mesurée $(\mathrm{V}), a$ le gain théorique $\left({ }^{\circ} \mathrm{C} / \mathrm{V}\right)$ et $b$ l'offset théorique $\left({ }^{\circ} \mathrm{C}\right)$. $L^{\prime}$ étendue de mesure est donnée par le convertisseur de $0{ }^{\circ} \mathrm{C}$ à $+400{ }^{\circ} \mathrm{C}(\mathrm{cf} . \S 3.1)$. Le convertisseur de température est utilisé avec une sortie en courant en $0-10 \mathrm{~V}$ (cf. $\S 3.1$ ). Donc $0{ }^{\circ} \mathrm{C}$ correspond à $0 \mathrm{~V}$ et $+400{ }^{\circ} \mathrm{C}$ correspond à $10 \mathrm{~V}$. On obtient un système de deux équations qui donne $a=40^{\circ} \mathrm{C} / \mathrm{V}$ et $b=0{ }^{\circ} \mathrm{C}$. D'où la loi affine : $y=40 x$.

Pour l'aspect expérimental, lorsque le mode «soudure froide à compenser » est choisi, le fabricant préconise d'utiliser une source de tension précise. Il faut alors brancher cette source de tension aux bornes 1 et 2 (figure 3). Pour régler l'offset, on doit appliquer une tension de $39 \mu \mathrm{V}$ et obtenir $25 \mathrm{mV}$ en sortie entre les bornes 7 et 8 (figure 3 ). Pour régler le gain, on doit appliquer une tension de $16,34 \mathrm{mV}$ et obtenir en sortie $10 \mathrm{~V}$.

\subsection{Programmation de la Chaîne de Mesure}

La programmation de l'API s'effectue directement depuis le PC. Elle est effectuée par logigramme.

Le fonctionnement d'un API, la modélisation d'une tâche et les modes de programmation d'un API font l'objet de cours / TD / TP effectués auparavant. Il s'agit donc ici d'un travail secondaire mais intéressant pour les liens avec les chaînes de mesure.

Le programme réalisé correspond au logigramme suivant :

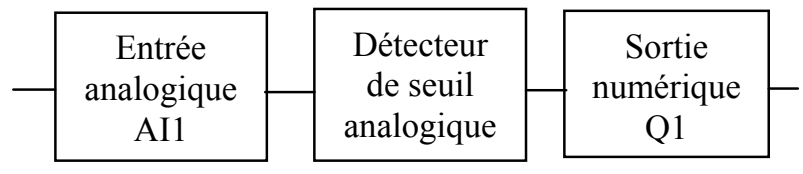

L'entrée analogique à choisir dépend des branchements réalisés. AI1 correspond au connecteur I7 sur l'API.

La fonction «Détecteur de seuil analogique » est une fonction prédéfinie. Elle a été choisie car acceptant une entrée analogique, capable de faire une conversion et disposant d'une sortie numérique. En effet, nous souhaitons voir la température et non des volts et l'API ne dispose que de sorties numériques. La fonction doit être paramétrée avec :

- la loi de conversion - tension lue par l'API en mesurande - n'est autre que la loi affine déterminée au paragraphe 3.2 ;

- les seuils de détection réglant l'état de Q1.

\subsection{Etude Métrologique de la Chaîne de Mesure}

Comme au paragraphe 2.3 , l'étude métrologique se limite à la détermination de l'incertitude-type de type B. Les sources d'erreur recensées sont :

- précision du thermocouple type $\mathrm{K}$ : classe 2 soit $\pm 2,5{ }^{\circ} \mathrm{C}$ de 0 à $330{ }^{\circ} \mathrm{C}$ (IEC 751);

- précision du convertisseur : précision de $1 \%$; erreur de transmission : $1,2 \%$ de la déviation $\max$. (ici $10 \mathrm{~V}$ ); erreur $\max$ due à la soudure froide : $\pm 4{ }^{\circ} \mathrm{C}$;

- précision du CAN de l'entrée analogique de l'API : 1000 valeurs sur $0-10 \mathrm{~V}$.

L'analyse est intéressante comparativement au précédent TP. Malgré une étendue de mesure supérieure -

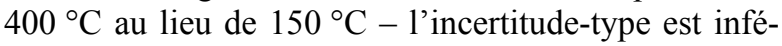


rieure : $6,4{ }^{\circ} \mathrm{C}$ au lieu de $13{ }^{\circ} \mathrm{C}$, à température ambiante $\left(22^{\circ} \mathrm{C}\right)$. La principale raison de cette importante différence résulte de la relative homogénéité en terme de performance de cette chaîne de mesure par rapport à la précédente chaîne. Trois sources d'erreur se partagent $95 \%$ de l'erreur totale : le thermocouple (25\% environ), l'erreur de transmission du convertisseur (35\% environ) et l'erreur due à la soudure froide (35\% environ). La précision du convertisseur et du CAN de l'API engendrent ici des erreurs négligeables par rapport aux autres sources. On retrouve le fait que le thermocouple est moins précis que le $\mathrm{Pt} 100$ et beaucoup plus délicat à mettre en œuvre à cause du problème de la compensation de la soudure froide. Enfin, cette fois, l'attention du métrologue doit surtout porter sur le convertisseur de température (qualité de la compensation de la soudure froide, qualité du transmetteur) et sur le choix du thermocouple (classe de précision).

\subsection{Utilisation de la Chaîne de Mesure : Vérification} Métrologique d'un Thermomètre

On dispose d'un $\mathrm{pH}$-mètre qui intègre une fonction température. Il se présente sous la forme d'un instrument de mesure complet de telle sorte que les différents éléments de la chaîne de mesure sont intégrés dans un même boîtier, hormis le transducteur qui se connecte au boîtier par une fiche BNC. Dans les laboratoires, cette solution est préférée aux chaînes de mesure " éclatées » comme celles présentées dans ces TPs pour :

- des mesurages ponctuels ;

- $\quad$ son ergonomie ;

- $\quad$ sa facilité d'utilisation ;

- etc.

En contrepartie, cette solution est moins intéressante pour :

- des mesurages automatiques ;

- des vérifications et / ou étalonnage d'éléments sur site ;

- la maintenance ;

- etc.

La méthode standard est la comparaison des résultats de mesurage fournis dans les mêmes conditions par l'instrument à vérifier - la fonction température du $\mathrm{pH}$ mètre - et ceux fournis par une chaîne de mesure étalon - la chaîne de mesure avec le thermocouple et l'API. On plonge le thermocouple et la sonde température dans un bain thermostaté à différentes températures couvrant le domaine d'utilisation prévu pour l'instrument.

\section{CONCLUSION}

Ces deux TPs forment un ensemble de fin d'unité d'enseignement. Ils permettent aux étudiants de remettre toutes leurs connaissances en perspective par rapport à des manipulations proches des réalités du terrain. Chaque étape comporte son lot de difficultés. En fonction de la formation initiale de l'étudiant, le montage des chaînes de mesure est plus ou moins évident. Le choix du matériel paraît simple pour tout le monde de prime abord dans la mesure où le nom des matériels est souvent explicite (convertisseur de température pour thermocouple par exemple). En réalité, la compréhension des fonctionnalités des différents matériels est délicate ; par exemple, le lien entre l'offset et le gain pour les convertisseurs et les résultats de mesurage reste abstrait tant qu'ils ne sont pas abordés en TP. Il s'ensuit des difficultés pour le calibrage des chaînes de mesure. De même, le lien entre la tension ou le courant fourni par un transmetteur et le résultat de mesurage n'apparaît clairement qu'avec la pratique. Les étudiants ne font le rapport entre mesurande - gain / offset - valeur transmise qu'après utilisation de la même loi dans les divers contextes. Le calcul d'une incertitude-type est d'ailleurs un contexte intéressant de ce point de vue. Cet exercice est malheureusement difficile pour tous les étudiants, notamment à cause des mathématiques utilisées. Enfin, les manipulations «utilisation de la chaîne de mesure ... » rapprochent dans la continuité ces TPs des tâches liées à la métrologie auxquelles les étudiants seront confrontés dans leur exercice professionnel.

\section{Bibliographie}

1.AFNOR NF X 07 - 001 Vocabulaire International des Termes Fondamentaux de Métrologie. 1994.

2. Asch, G and Al. Les capteurs en instrumentation industrielle. Dunod, 3ème édition, 1987, pp. 1-12. 